\title{
Allergens Are Not Detected in the Bronchoalveolar Lavage Fluid of Patients Undergoing Fiberoptic Bronchoscopy
}

Rueda $\mathrm{M}^{1}$, López-Matas $\mathrm{MA}^{2}$, Agustí $\mathrm{C}^{3}$, Lucena $\mathrm{C}^{3}$, Carnés $\mathrm{J}^{2}$, Valero $\mathrm{A}^{3,4}$

${ }^{1}$ Allergology Service, Hospital Quirónsalud, Barcelona, Spain

${ }^{2} R \& D$ Department, Laboratorios LETI S.L.U., Tres Cantos, Madrid, Spain

${ }^{3}$ Neumology and Allergy Service, Hospital Clinic, Barcelona, Spain

${ }^{4}$ Institut d'Investigacions Biomèdiques August Pi i Sunyer (IDIBAPS), Centro de Investigaciones Biomédicas en Red de Enfermedades Respiratorias (CIBERES), Barcelona, Spain

J Investig Allergol Clin Immunol 2019; Vol. 29(2): 148-150 doi: $10.18176 /$ jiaci. 0353

Key words: Bronchoalveolar lavage. Allergen. Mass spectrometry. Monoclonal Antibodies. Small airway.

Palabras clave: Lavado broncoalveolar. Alérgeno. Espectrometría de masas. Anticuerpos monoclonales. Vías aéreas inferiores.

Allergen sources range in size from 0.28 to $0.40 \mathrm{~mm}$ for mites, 2 to $500 \mu \mathrm{m}$ for molds, and 2 to $200 \mu \mathrm{m}$ for pollens. Early studies suggested that given their large size, aeroallergenic sources were unable to reach the lower airways [1]. The nose filtering system retains particles larger than $10 \mu \mathrm{m}$, and only fine or ultrafine particles reach distal bronchioles (diameter 1-5 $\mu \mathrm{m}$ ) [2,3]. However, it has been demonstrated that grass pollen allergens are released as breathable aerosols, the atmosphere contains allergencarrying, plant-derived, paucimicronic particles $(2-5 \mu \mathrm{m})$ [4], and small spores and fragments of mold spores can reach the lower airways [5].

Ferguson et al [6] identified the major allergen from Dermatophagoides pteronyssinus (Der p 1) in the small airways of allergic patients. The authors assessed the link between environmental exposure to mites and the presence of the allergen in bronchoalveolar lavage (BAL) fluid in 9 patients sensitized to D pteronyssinus.

Horvath et al [7] studied deposition of inhaled pollens in the airway using computer simulation. Their results suggested that pollen particles $(0.5-20 \mu \mathrm{m})$ may deposit more efficiently in the asthmatic lung than in the healthy lung, especially in the bronchial region.

The aim of our study was to examine the possible presence of major allergens from mites, pollen, and molds in BAL fluid collected from patients undergoing fiberoptic bronchoscopy.

We performed an observational descriptive study. BAL samples were obtained from patients undergoing fiberoptic bronchoscopy (hospitalized and outpatients) over 1 year. Indications for fiberoptic bronchoscopy included hemoptysis, chronic cough, and upper airway assessment. Patients with 
asthma were also included. The study protocol was approved by the Ethics Committee of Hospital Clínic, Barcelona, Spain. Fiberoptic bronchoscopy with BAL was performed following the recommendations of the Spanish Respiratory Society. Briefly, lavage consisted of infusing 3 aliquots ( $50 \mathrm{~mL}$ each) of sterile saline solution into 1 of the subsegments of the middle lobe or lingula. The first aliquot was collected for this study and stored at $-80^{\circ} \mathrm{C}$ until analysis. BAL samples were dialyzed and lyophilized to avoid loss of sensitivity due to dilution. For protein profiling, $100 \mu \mathrm{g}$ of samples lyophilized before and after dialysis were run on SDS-PAGE. The major allergens Phl p 5, Alt a 1, and Der p 1 were quantified using an ELISA sandwich assay (Indoor Biotechnologies). Two samples were analyzed using mass spectrometry (Orbi-LTQ Velos-Pro) and compared with the peptide databases Homo, Viridiplantae, Fungi, and Arthropoda using the software Proteome Discoverer 1.3 (Proteomic Service, CBMSO, Madrid, Spain).

BAL samples were collected from 48 patients. The reasons for bronchoscopy were assessment of noninfectious pulmonary conditions, mainly bronchial neoplasias (13 patients), noninfectious lung infiltrates (17 patients), and interstitial lung disease ( 6 patients). The indications for bronchoscopy in the remaining 12 patients were chronic cough, bronchiectasis, acute respiratory distress syndrome, asthma, chronic obstructive pulmonary disease, allergic bronchopulmonary aspergillosis, and amyloidosis.

Protein profiles were similar for all tested samples. The main differences observed between samples were in their protein quantity. All samples showed 2 main bands at 50-60 $\mathrm{kDa}$, and 3 at 30,21 , and $15 \mathrm{kDa}$ (Figure). None of the allergens tested (Phl p 5, Alt a 1, Der p 1) were detected using the ELISA kits. Mass spectrometry enabled 2684 and 6139 peptides to be recovered from each sample respectively. No peptides from mites, plants, or fungi were detected after comparison of the sequences with those provided in the databases. More than $99 \%$ of the peptides were components of human proteins. The remaining peptides were homologous to unknown or conserved proteins such as actin and tubulin, neither of which was reported as an allergen.

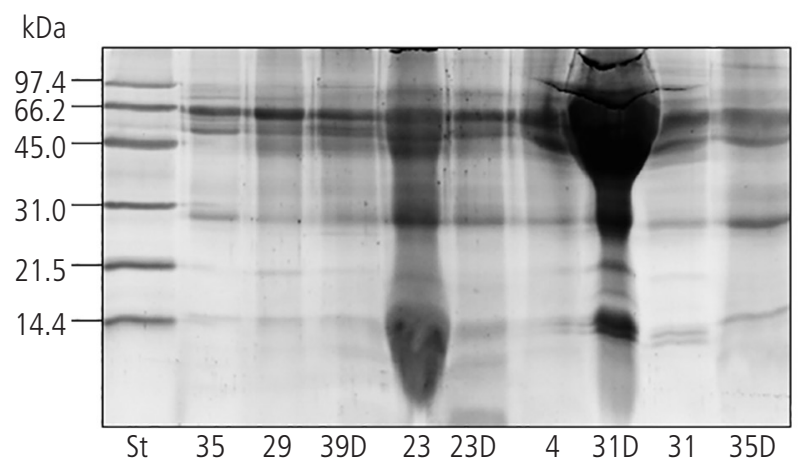

Figure. SDS-PAGE with a selection of samples. One hundred micrograms of lyophilized powder was run in each lane. The numbers under each lane correspond to the number of the patient sample. Lines with numbers only correspond to predialysis samples, and lines with numbers followed by a D correspond to postdialysis samples. St indicates molecular weight standard low-range (Bio-Rad).
Our study was designed to address the presence of allergens in the small airways by examining BAL samples obtained from patients undergoing bronchoscopy to assess various respiratory conditions. BAL is a bronchoscopic technique commonly used to diagnose lung diseases. For research purposes, this technique is also used to sample epithelial lining fluid components and to determine the protein composition of airway content. The hypothesis was that if major allergens were detected in BAL, then they would indicate the capacity of these particles to reach the small airways. However, none of the tested allergens were detected in the BAL samples.

The presence of Der $p 1$ [4] has been described in BAL from asthma patients, although it is not known whether this allergen occurs in the general population. As far as we know, this is the first study to address the presence of major allergens in the small airways of the general population. Although, our study should ideally have included healthy individuals, the number of patients examined and their heterogeneous nature suggest that this sample could well reflect the general population. The patients lived in a non-allergen-free environment. In our area, the allergens evaluated are mainly seasonal, with maximum incidences in specific seasons. House dust mites peak in spring and autumn [8], fungal spores in summer and autumn [9], and grass pollen in spring and summer. To ensure the presence of allergens in the environment, we collected BAL samples over a whole year.

Analyzing BAL is technically challenging, thus partially explaining why we were unable to identify airborne antigens. Different particles, especially high-molecular-weight molecules, can be lost during the liquid concentration process. Besides, not knowing the dilution of alveolar fluid in the saline instilled makes it difficult to properly determine extracellular particles. Finally, low-molecular-weight compounds quickly diffuse from the alveoli to the interstitium and capillaries. Despite these limitations, our methodology, which included the use of monoclonal antibodies and mass spectrometry, ensured the validity of our results. Mass spectrometry mainly detected human proteins from epithelial cells entering the lavage fluid. Other peptides corresponded to very well-conserved proteins, and differences were probably due to small variations in mass detection. Although we identified some of these peptides, none of them were constituents of known allergens.

In summary, we were not able to detect major allergens of mites, pollen, or molds in BAL. Further studies are needed to assess potential differences in detected allergens between populations, mainly asthma patients and those with other lung diseases. This type of information will help establish whether or not it is necessary for an allergen to reach the distal airways to trigger an asthma attack.

\section{Funding}

This study was funded by Societat Catalana d'Al·lèrgia.

\section{Conflicts of Interest}

MA López-Matas and J Carnés are employees of Laboratorios LETI. The remaining authors declare that they have no conflicts of interest. 


\section{References}

1. Shah R, Grammer LC. Chapter 1: an overview of allergens. Allergy Asthma Proc. 2012;33 Suppl 1:S2-5.

2. Wilson AF, Novey HS, Berke RA, Surprenant EL. Deposition of inhaled pollen and pollen extract in human airways. N Engl J Med. 1973;288:1056-8.

3. Cheng Y, Smith SM, Yeh H. Deposition of ultrafine particles in human tracheobronchial airways. Ann Occup Hyg. 1997;41:714-8.

4. Taylor PE, Flagan RC, Valenta R, Glovsky MM. Release of allergens as respirable aerosols: A link between grass pollen and asthma. J Allergy Clin Immunol. 2002;109:51-6.

5. Rick EM, Woolnough K, Pashley $\mathrm{CH}$, Wardlaw AJ. Allergic Fungal Airway Disease. J Investig Allergol Clin Immunol. 2016;26(6):344-54.

6. Ferguson P, Broide DH. Environmental and bronchoalveolar lavage Dermatophagoides pteronyssinus antigen levels in atopic asthmatics. Am J Respir Crit Care Med. 1995;151:71-4.

7. Horvath A, Balashazy I, Farkas A, Sarkany Z, Hofmann W, Czitrovszky A, et al. Quantification of airway deposition of intact and fragmented pollens. Int J Environ Heat R. 2011;21:427-40.

8. Demoly P, Matucci A, Rossi O, Vidal C. A year-long, fortnightly, observational survey in three European countries of patients with respiratory allergies induced by house dust mites: Methodology, demographics and clinical characteristics. BMC Pulm Med. 2016;16:85.

9. Gomez de Ana S, Torres-Rodríguez JM, Ramírez EA, García SM, Belmonte-Soler J.J. Seasonal distribution of Alternaria, Aspergillus, Cladosporium and Penicillium species isolated in homes of fungal allergic patients. J Investig Allergol Clin Immunol. 2006;16:357-63.

Manuscript received October 17, 2018; accepted for publication November 14, 2018.

Jerónimo Carnés Research \& Development Laboratorios LETI, S.L.U. E-mail: jcarnes@leti.com 